\title{
Homogeneous nucleation in spatially inhomogeneous systems
}

G. Shi and J. H. Seinfeld

Department of Chemical Engineering, California Institute of Technology, Pasadena, California 91125

K. Okuyama

Department of Chemical Engineering, University of Osaka Perfecture, Sakai, Osaka 591 Japan

(Received 5 March 1990; accepted for publication 9 July 1990)

Homogeneous nucleation of a vapor in the presence of the loss of clusters by diffusion and thermophoretic drift is investigated. Analytical results are obtained for the cluster size distribution and the rate of nucleation by solving the modified kinetic equation for nucleation. The implications of cluster loss by diffusion and phoretic drift on the onset of the homogeneous nucleation of silicon vapor in the horizontal epitaxial chemical vapor deposition reactor is discussed. The range of conditions under which the loss of subcritical clusters by diffusion and drift becomes important for the interpretation of diffusion cloud chamber experimental data of the onset conditions of the homogeneous nucleation of vapors is also delineated.

\section{INTRODUCTION}

Many systems in which nucleation occurs are spatially nonuniform, and nucleation occurs only in those regions in which the supersaturation exceeds the critical value. Subcritical clusters formed within this region may leave it by diffusion or by drift under the influence of an external potential. Such diffusional and drift loss processes compete with the growth of clusters to the critical size and may reduce the rate of nucleation below that predicted by conventional nucleation theories in which the subcritical cluster loss is not included. The goal of the present work is to extend classical homogeneous nucleation theory to include subcritical cluster loss by diffusion and phoretic drift. The implications of subcritical cluster loss by diffusion and phoretic drift to the possible control of the onset of nucleation of silicon particles in a horizontal epitaxial chemical vapor deposition (CVD) reactor is discussed in light of the present theoretical results. We will also investigate the implications of subcritical cluster loss by diffusion and thermophoretic drift to the interpretation of experimental data from the diffusion cloud chamber which is often employed to study the onset conditions of homogeneous nucleation.

\section{FORMULATION OF THE PROBLEM}

Consider a cluster containing $g$ monomers, within a onedimensional region of thickness $d_{0}$. Such a cluster may grow or evaporate or diffuse or drift out of the region. We consider the cluster size $g$ as a continuous variable. Then the cluster distribution function $f(g, z, t)$ at position $z$ obeys the continuity equation:

$$
\frac{\partial f(g, z, t)}{\partial t}+\frac{\partial j(g, z, t)}{\partial g}+\frac{\partial h(g, z, t)}{\partial z}=0,
$$

where we have introduced the fluxes of clusters in the cluster size space, $j(g, z, t)$, and in the physical space, $h(g, z, t)$. The flux $h(g, z, t)$ is assumed to include contributions from Brownian diffusion and thermophoretic drift,

$$
h(g, z, t)=-D(g) \frac{\partial f}{\partial z}-V_{T} f
$$

where $D(g)$, the Brownian diffusion coefficient for a $g$-sized cluster, is given by'

$$
D(g)=\frac{12 \pi(k T)^{2 / 3} g^{2 / 3}}{8 p_{i} \sqrt{2 \pi m_{c}} s_{1}}
$$

with $m_{\mathrm{r}}$ the mass of a carrier gas molecule which is assumed smaller than that of a monomer; and $V_{T}$ is the thermophoretic drift velocity for a cluster of free molecule size,

$$
V_{T}=-\frac{3 v}{4} \frac{d \ln T}{d z}
$$

Here $v$ is the kinematic viscosity of the carrier gas.

An expression for the flux of the clusters in the cluster size space $j(g, z, t)$ can be written by allowing for the fact that the number concentration of clusters is substantially smaller than the number concentration of monomers, so that the motion along $g$ occurs mainly through the interaction of clusters with monomers. Then the expression for the flux

$j(g, z, t)$ is ${ }^{2}$

$$
j=-\beta n \frac{\partial}{\partial g} \frac{f}{n},
$$

where $n(g, z)$ is the equilibrium cluster distribution and

$$
\beta=n(1) s_{1} g^{2 / 3}\left(k T / 2 \pi m_{1}\right)^{1 / 2}
$$

with $s_{1}$ and $m_{1}$ the surface area and mass of the monomer, respectively. According to the capillary approximation, $n(g, z)$ can be written as ${ }^{2}$

$$
n(g, z)=n(1, z) \exp \left(g \ln S-\theta g^{2 / 3}\right),
$$

where $\theta$ is a dimensionless surface tension,

$$
\theta=s_{1} \sigma / k T
$$

and where the saturation ratio $S=n(1, z) / n_{\mathrm{eq}}, \sigma$ is the surface tension of the particle, and $n_{\mathrm{c} y}(1)$ is the equilibrium number concentration of the monomer. The capillary approximation will be employed because we are only interested in the conditions for the onset of homogeneous nucleation of vapors. At low rates of nucleation, it is assumed that the capillary approximation may be invoked even for the nucleation of silicon vapor at high temperatures. Boundary condi- 
tions are specified at two physical boundaries on $z$ besides the usual boundary conditions on $g: f / n=1$, as $g \rightarrow 1$ and $f / n=0$, as $g \rightarrow \infty$. Profiles that might be achieved in a diffusion cloud chamber and a CVD reactor are schematically shown in Figs. 1(a) and 1(b), respectively as examples of systems in which nucleation is confined to a thin spatial region.

The governing equations can be simplified if the saturation ratio is sufficiently large in the narrow region of thickness $d_{0}$ where nucleation occurs. The saturation ratio decreases rapidly on either side of this region, and since the concentration of clusters depends strongly on the supersaturation, virtually no clusters can be assumed exist on either side of the region. If the cluster concentrations at the two physical boundaries at distances $d_{1}$ and $d_{2}$ from the thin region $d_{0}$ are assumed to be zero, the gradients for diffusion toward the two boundaries can be estimated by $f / d_{1}$ and $f / d_{2}{ }^{2}$ Then the diffusion loss of $g$-sized clusters per unit time from a unit area will be

$$
D(g)\left(\frac{1}{d_{1}}+\frac{1}{d_{2}}\right) f
$$

Since the region of nucleation has thickness $d_{0}$, the loss per unit time per unit volume will be $D(g)\left(d_{0} d\right)^{-1} f$, where

$$
\frac{1}{d}=\frac{1}{d_{1}}+\frac{1}{d_{2}}
$$
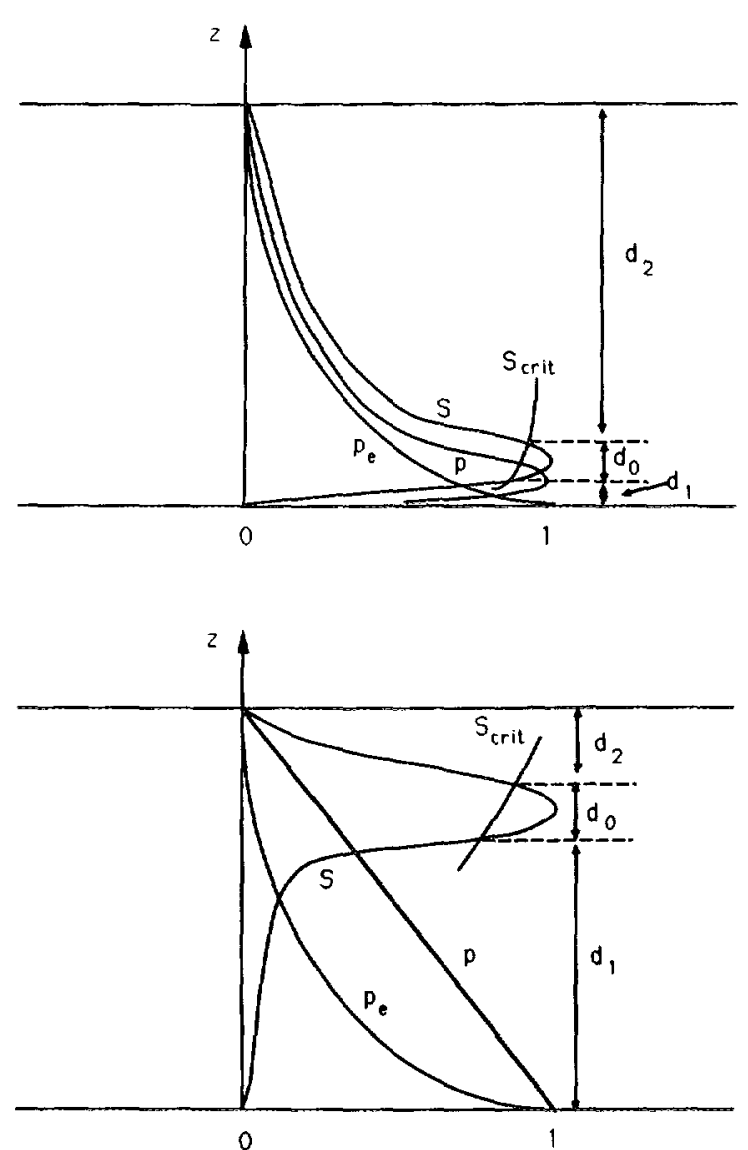

FIG. 1. Schematic profiles in a nonuniform nucleation system: (a) diffusion cloud chamber; (b) CVD reactor.
The term $V_{r} \partial f / \partial z$ describing the subcritical cluster loss by thermophoretic drift per unit time per unit volume will be approximated as $V_{T} f / d_{2}$ since clusters driven by the thermopheretic force will move to the upper surface when the temperature gradient is assumed to be along the $z$ direction. The gradient set up in this manner will underestimate the actual value since a subcritical cluster (which is larger than the critical size in the region $d_{0}$ ) will decompose back to monomer immediately outside the region $d_{0}$. With these simplifications and assuming a stationary state, Eq. (1) with Eq. (2), becomes within the region of nucleation

$0=-\frac{\partial j(g)}{\partial g}-D(g) \frac{1}{d}\left(\frac{3}{2} \omega+\frac{1}{d_{0}}\right) f-\omega \frac{3 v}{4}\left(\frac{1}{d_{2}}+\omega\right) f$

where $\omega=d \ln T / d z$

To obtain the critical conditions for the onset of homogeneous nucleation and its rate, it is necessary to determine the cluster size distribution by solving the above equation. The procedure based on matched asymptotic expansions developed by Shi and Seinfeld ${ }^{3}$ will be followed in solving Eq. (9).

\section{CLUSTER DISTRIBUTION FUNCTION AND NUCLEATION RATE}

\section{A. Cluster distribution function}

Introducing $y=f / n$, and $x=g / g_{*}$, Eq. (9) becomes

$\epsilon^{2} \frac{\partial^{2} y}{\partial x^{2}}+\left(\frac{2}{3 x} \epsilon^{2}+6\left(1-x^{1 / 3}\right)\right) \frac{\partial y}{\partial x}$

$$
=2\left(a x^{4 / 3}+b x^{2 / 3}\right) y,
$$

where $\epsilon \equiv \delta / g_{*}$ with $\delta$ and $g_{*}$ given by

$$
\delta=3 g_{*}^{2 / 3} \theta^{1 / 2}, \quad g_{*}=\left(\frac{2 \theta}{3 \ln S}\right)^{3} .
$$

Here $g_{*}$ is the critical cluster size above which a cluster grows spontaneously. $\delta$ defines the width of the critical region about $g_{*}$ such that the difference between the Gibbs formation energy of clusters at size $g$ and those at $g_{*}$ is smaller than $k T$. Also, in Eq. (10),

$$
\begin{aligned}
& a=\frac{\delta^{2}}{2 \beta\left(g_{*}\right)}\left(\frac{1}{d_{0}}+\frac{3 \omega}{2}\right) \frac{D\left(g_{*}\right)}{d}, \\
& b=\frac{\delta^{2}}{2 \beta\left(g_{*}\right)}\left(\frac{1}{d_{2}}+\omega\right) \frac{3 \omega v}{4}
\end{aligned}
$$

are two dimensionless time scales. $a$ is the ratio between the characteristic nucleation relaxation time ${ }^{4}$ which is given by $\delta^{2} / 2 \beta\left(g_{*}\right)$ and that of Brownian diffusion of clusters. Thus, a large value of $a$ implies that the response time of the nucleation process to changes in the saturation ratio is longer than the time it takes for clusters to diffuse out of the region of thickness $d_{0}$. On the contrary, if $a$ is small, the response time of the nucleation process to changes in the saturation ratio is shorter than the time it takes for clusters to diffuse out of the regions of thickness $d_{0} . b$ is the ratio of the characteristic nucleation relaxation time to that for the thermophoretic drift of clusters. Thus a large value of $b$ implies that the nucleation process responds slowly to changes in the satura- 
tion ratio relative to the time it takes for clusters to thermally drift out of the region of thickness $d_{1}$, and vice versa.

To a leading-order approximation in $\epsilon$ the nonequilibrium cluster distribution function obtained by the method of matched asymptotic expansions is

$$
\begin{aligned}
y(x)= & \left(\frac{x^{1+3}-1}{g_{*}^{1 / 3}-1}\right)\left(\frac{1-x^{1 / 3}}{1-g_{*}^{1 / 3}}\right)^{b} \exp \left[b\left(x^{1 / 3}-g_{*}^{-1 / 3}\right)\right] \\
& +\frac{1}{2} A i^{a+b} \operatorname{erfc}\left(\frac{x-1}{\epsilon}\right) \\
& -A \Gamma^{\prime}(a+b+1)\left(\frac{1-x}{\epsilon}\right)^{a+b}
\end{aligned}
$$

where

$$
\begin{aligned}
A= & \left(\frac{\delta}{3 g_{*}}\right)^{a, h} \Gamma(a+b+1)\left(g_{*}^{1 / 3}-1\right) \quad{ }^{a}\left(1-g_{*}^{1 / 3}\right) \cdot b \\
& \times \exp \left[b\left(1-g_{*}^{1 / 3}\right)\right]
\end{aligned}
$$

and $i^{\prime \prime} \operatorname{erfc}(x)$ is an integrated error function, and $\Gamma \quad '(x)$ is the inverse of the Gamma function, $\Gamma(x)$.

In the limit of $a=b=0$, Eq. (12) reduces to the correct limiting result for the cluster size distribution in a spatially uniform system,"

$$
f(g)=\frac{1}{2} n(g) \operatorname{erfc}\left(\frac{g-g_{*}}{\delta}\right) .
$$

$y(x)$ is shown in Fig. 2 for $a=0, b=0$, the spatially uniform case, and $a=1,3,5, b=0$. It can be seen from Fig. 2 that both the concentration of the critical sized cluster and the slope of $f / n$ at $g_{*}$, which is related to the rate of crossing at $g_{*}$ [see Eq. (16) below] significantly reduced in the presence of subcritical cluster diffusion $(a \neq 0)$ compared with that in its absence. These two factors directly contribute to the reduction in the rate of nucleation.

\section{B. Nucleation rate}

The nucleation rate in the presence of subcritical cluster loss by diffusion and thermophoretic drift is given by the flux of clusters in the size space past the critical size $g_{*}$,

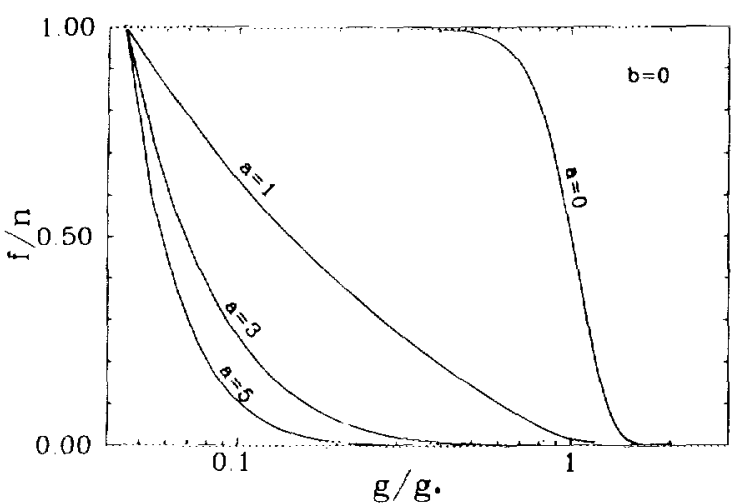

FIG. 2. Steady-state nonequilibrium cluster size distributions in the case of subcritical cluster losis by diffusion.

$$
\begin{aligned}
j\left(g_{*}\right) & =-\left.\beta(g) n(g) \frac{\partial}{\partial g} \frac{f(g)}{n(g)}\right|_{g:-g_{*}} \\
& =j_{u} A \sqrt{\pi 2} \quad{ }^{(a+b)} \Gamma \quad\left(\frac{a+b+1}{2}\right),
\end{aligned}
$$

where

$$
j_{u}=\beta\left(g_{*}\right) n\left(g_{*}\right)(1 / \sqrt{ } \pi \delta)
$$

is the rate of homogeneous nucleation in the absence of subcritical cluster loss but with other conditions being the same as in the inhomogeneous system (i.e., the same $T$ and $S$ ). As expected, $j\left(g_{*}\right)$ reduces to $j_{u}$ when $a=b=0$, and the ratio $j\left(g_{*}\right) / j_{u}$ decreases with increasing values of $a$ and $b$. It is expected that $j\left(g_{*}\right) / j_{u}$ tends to zero as $a$ and/or $b \rightarrow \infty$. Eq. (16), however, does not produce this required limit. In fact the leading-order perturbation requires that $a<3 / \epsilon$ and $b<3 / \epsilon$. Thus, Eq. (16) underestimates the effect of subcritical cluster diffusion on the rate of nucleation for $a>3 / \epsilon$ and/or $b>3 / \epsilon$. (In figures presented below, we will indicate the calculations beyond these ranges by dashed lines.) A higher-order approximation in $\epsilon$ will give a solution that is valid over a wider range of $a$ and $b$. For the present purpose of studying the onset of homogeneous nucleation, it is sufficient to have this leading-order solution.

\section{NUCLEATION IN SPATIALLY INHOMOGENEOUS SYSTEMS}

\section{A. Particle formation in the horizontal epitaxial CVD reactor}

The pyrolysis of silane $\left(\mathrm{SiH}_{4}\right)$ is widely used in epitaxial growth of silicon film. ${ }^{6-16}$ Figure 1 (a) indicates profiles that might be achieved in a CVD reactor in which a silicon compound is deposited from the gas phase onto a hot substrate. The reaction zone in which silicon atoms are formed from silane decomposition will be localized in a thin region near the hot surface. ${ }^{6,9,10,13}$ Most of the silicon atoms will then diffuse to the substrate and undergo condensation to form a film. The higher deposition rates correspond to higher diffusion rates which can be achieved by steepening the concentration gradient at the interface, by increasing the silane partial pressure and by reducing the diffusion path length (reducing the total gas pressure).$^{6-8}$ An increase in silane concentration, however, does not always give a proportional increase in the deposition rate. There is a competing process which can cause nucleation of silicon vapor to form silicon particles in the gas phase resulting in a loss of silane and thus a reduction of silicon deposition rate, when the concentration of silicon atoms exceed the critical saturation ratio. Particle formed in CVD generally have a deleterious effect on film quality. At low gas temperatures the onset of gas-phase nucleation can probably be prevented, but to get a good crystalline quality of the epi-layer, a high substrate temperature ( $1050{ }^{\circ} \mathrm{C}$ or above) is usually required. ${ }^{\mathrm{b}, ?}$

Most of the work related to the onset of homogeneous nucleation of silicon vapor in epitaxial reactors has been experimental. Conventional nucleation theory has been used to investigate the temperature dependence of the nucleation rate of silicon vapor. ${ }^{16}$ However, the main complexity in the 
use of nucleation theory for such calculations is the great nonuniformity of the process when the subcritical cluster escapes as discussed in the introduction. With the necessary theoretical formulation developed above, we will investigate the implications of subcritical cluster loss by diffusion and drift on prevent from the onset of homogeneous nucleation of silicon vapor in epitaxial reactors.

First let us consider a case with the gas temperature of $1027^{\circ} \mathrm{C}$ at the nucleation zone in a reactor charged with $\mathrm{H}_{2}$ as a carrier gas of atmosphere pressure. Following classical nucleation theory, ${ }^{16}$ and use the available data for the equilibrium vapor-solid pressure, the critical saturation ratio is 26.4 in the absence of subcritical cluster loss. At this otherwise critical saturation ratio $S=26.4, a$ is about $12.84 \phi$ and $b$ is $0.15 \psi$ and the dependence of the ratio $j\left(g_{*}\right) / j_{u}$ on $a$ and $b$ is shown in Figs. 3(a) and 3(b). Here

$$
\begin{aligned}
& \phi=\frac{1}{d}\left(\frac{1}{d_{0}}+\frac{3 \omega}{2}\right), \\
& \psi=3 \omega\left(1 / d_{2}+\omega\right), \\
& a=\frac{4 \pi}{9 p_{1}} \sqrt{\frac{m_{1}}{m_{r}}}\left(\frac{k T}{s_{1}}\right)^{3} \frac{\phi}{p_{e} S \sigma},
\end{aligned}
$$

and

$$
b=\frac{2 \sigma}{p_{c} p_{t} S \ln ^{2} S} \sqrt{2 \pi \frac{m_{1}}{k T}} \frac{v R_{g} T \psi}{M_{c}}
$$
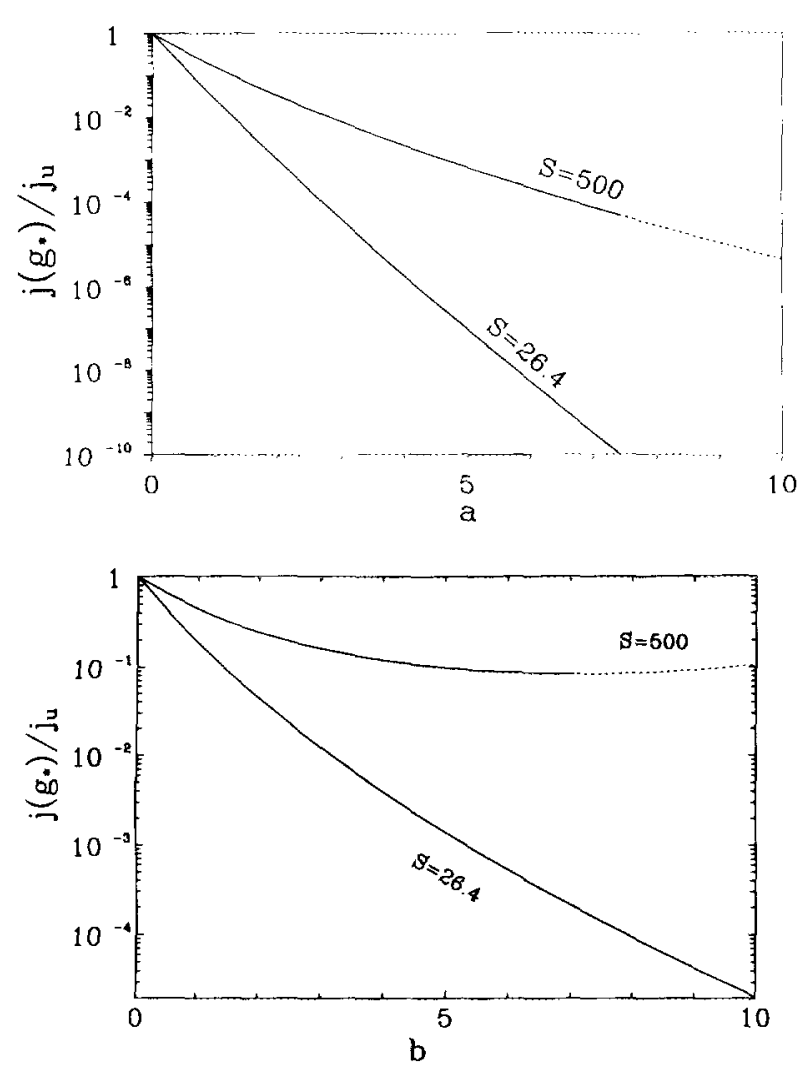

FIG. 3. Ratio between the rate of nucleation in presence of subcritical cluster loss in a horizontal epitaxial CVD reactor and that in its absence (a) by diffusion $\left[j\left(g_{*}\right) / j_{k}\right.$ vs $a$ for $\left.b=0\right]$ and (b) by thermophoretic drift $\left[j\left(g_{*}\right) / j_{k}\right.$ us $b$ for $\left.a=0\right]$. where the total pressure in the reactor is assumed mainly due to the carrier gas and $M_{c}$ is the molecular weight of the carrier gas and $R_{y}$ is the universal gas constant.

For an atmospheric CVD reactor with $\mathrm{H}_{2}$ as the carrier gas, we can estimate $d_{1}=0.45 \mathrm{~cm}, d_{11}=0.3 \mathrm{~cm}, d_{2}=4.25$ $\mathrm{cm}$ based on available data. ${ }^{13}$ If we also take $d T / d z=1000^{\circ} \mathrm{C} / \mathrm{cm}$, we can obtain $\phi$ and $\psi$, thus $a$ and $b$. In this case, $a$ is about 142 and $b$ is 0.04 . From Eq. (15) the ratio $j\left(g_{*}\right) / j_{u}$ is virtually zero for $S=26.4$ Thus the onset of homogeneous nucleation of silicon vapor at this temperature is totally suppressed by the subcritical cluster loss due to the subcritical cluster loss by diffusion. Even at $S=500, a$ is about 7.5 and the ratio $j\left(g_{*}\right) / j_{u}$ is about $10^{10}$ while $j_{u}=10^{\circ} \mathrm{cm}{ }^{3} \mathrm{~s}{ }^{1}$. Thus the critical saturation ratio in the presence of subcritical cluster loss is about 20 times larger than that in its absence. The thermophoretic drift loss in both cases is negligible which is in contrast to previous suggestion. ${ }^{6.7}$ Thermophoretic drift becomes relatively important for larger particles and becomes at least equally important as subcritical cluster diffusion at temperature exceeding $T=1412^{\circ} \mathrm{C}$.

In the above calculations, it is clear the thermal structure in the CVD reactor which determines the values of $d_{0}$, $d_{1}$, and $d_{2}$ is central in determining the relative importance of subcritical cluster loss to the onset of nucleation. As observed by Breiland et al., ${ }^{13}$ the maximum $\mathrm{Si}$ is very close to the substrate within $2 \mathrm{~mm}$ in a cell of $5 \mathrm{~cm}$ height when the substrate temperatures is in the range of $710-1000^{\circ} \mathrm{C}$. As the substrate temperature increases, the thermal boundary layer extends farther above the surface. The temperature at which silane rapidly decomposes thus occurs at larger distances $\left(d_{1}\right)$ from the surface. Also the spatial range $\left(d_{0}\right)$ over which particles are produced increases with increasing temperature as shown by the broadening of the profiles of $\mathrm{Si}{ }^{1{ }^{13}}$ Thus at a higher temperature, we expect a smaller $\phi$ and a smaller $\psi$ and thus a smaller effect of subcritical cluster diffusion on the nucleation rate. For example at $T=1412{ }^{\circ} \mathrm{C}$, we take $d_{1}=1 \mathrm{~cm}, d_{0}=0.5 \mathrm{~cm}, d_{2}=3.5 \mathrm{~cm}$ based on observed trends. ${ }^{13}$ In this case $j\left(g_{*}\right) / j_{d}$ is 10 ' at the otherwise critical supersaturation ratio $S=16$. This calculation shows that the kinetic nucleation barrier due to subcritical cluster loss by diffusion is also higher at lower temperature. Since at relatively low operating temperatures, the subcritical clusters take more time to accumulate in the gas phase, it should be relatively easy to suppress the occurrence of possible nucleation by optimizing operating total pressure and temperatures and using a light gas as the carrier.

The effect of carrier gas on the onset of homogeneous nucleation of silicon vapor in the CVD reactor can be in part explained by the present theory. It is observed that all other conditions being equal $\mathbf{H}_{2}$ as a carrier gas suppresses particle formation compared to $\mathrm{He}$, which cannot be simply accounted for by the chemical kinetic effect of $\mathrm{H}_{2}$ on the gasphase decomposition of silane. "The carrier gas effect is in part explained by the fact $a$ is 1.414 times larger and $b$ is 1.114 times larger in $\mathrm{He}$ than in $\mathrm{H}_{2}$. It is noted that the effect of the possible changes in $d_{0}, d_{1}$, and $d_{2}$ due to different thermal structures in the $\mathrm{He}$ or $\mathrm{H}_{2}$ carrier gas is not able to be evaluated. 
According to Eqs. (17) and (18), $a$ and $b$ are inversely proportional to the total system pressure $p_{t}$ as the diffusion path lengths are inversely proportional to $p_{r}$. Thus a direct effect of reducing total pressure is to suppress the onset of homogeneous nucleation in the reactor. However, the position of the maximum in Si density in the reactor shifts up at a reduced pressure. It is observed that the position of this maximum shifts from $1 \mathrm{~mm}$ at atmosphere pressure to about 1 $\mathrm{cm}$ above the surface at a total pressure of about 20 Torr when the substrate temperat ure is $847^{\circ} \mathrm{C}^{1.3}$ The same experiment aiso showed that the profiles at the reduced pressure become much broader than at an atmosphere pressure. Thus particle formation could occur in a much wider region at the reduced total pressure. Although $a$ and $b$ increase by a factor of 35 when from the reducing the total pressure from 760 to 20 Torr the change in $d_{0}, d_{1}$, and $d_{2}$, could outweigh this reduction.

Purely lowering the total pressure may not prevent the onset of homogeneous nucleation if a sufficiently thick thermal boundary layer is attained to reduce the efficiency of subcritical cluster loss by diffusion. This may have been why particle formation in the gas phase was still observed at lower pressure CVD (LPCVD). ${ }^{910}$

It is clear that the thermal structure in the CVD reactor is very important in determining the efficiency of subcritical cluster diffusion towards the substrate. If the relationship between the values of $d_{0}$ and $d_{1}$ and the total gas pressure and the substrate temperature is known experimentally ${ }^{13}$ or by modelling, ${ }^{13}$ one can use Eqs. (16)-(18) to select the optimal CVD conditions in order to minimize the possibility of occurrence of silicon particle formation in the gas phase while obtaining a high deposition rate for a given silane concentration.

\section{B. Nucleation in diffusion cloud chambers}

The diffusion cloud chamber is one of the principal methods for studying conditions tor the onset of homogeneous nucleation. Supersaturation varies with elevation in a diffusion cloud chamber [Fig. 1(b)] and nucleation is observed in a thin region within the chamber. The possible effects of nonuniformities in the cloud chamber on the nucleation rate or the critical supersaturation ratio were suggested not to cause serious deviations from behavior in the absence of subcritical cluster loss for substances with equilibrium vapor pressures larger than $10{ }^{8}$ Torr. $^{2}$ It will be shown. however. that substantial deviations can be occurred for substances even with much higher equilibrium vapor pressures if the cloud chamber is operated at the reduced pressures. ${ }^{17}$

Consider a system with $\sigma=30$ dyne $\mathrm{cm}$, $s_{1}=1.5417 \times 10^{14} \mathrm{~cm}^{2}, m_{1}=1.6 \times 10^{22} \mathrm{~g}, T=20^{\circ} \mathrm{C}$ and $p_{t}=1$ atm with $\mathrm{He}$ as the carrier gas, ${ }^{13}$ $a=4.6 \times 10{ }^{8} \phi /(p, S)$, where $p$ is in Torr. As calculated earlier, ${ }^{2}$ the critical supersaturation in the absence of subcritical cluster loss is about 12.5 for $p_{c}=10^{-5}$ Torr, and the value of $a$ is thus about $10{ }^{+} \phi$. The change of the ratio $j\left(g_{*}\right) / j_{u}$ with $a$ and $b$ for these conditions is shown in Figs. $4(a)$ and $4(b)$. Thus, there is no effect of subcritical cluster
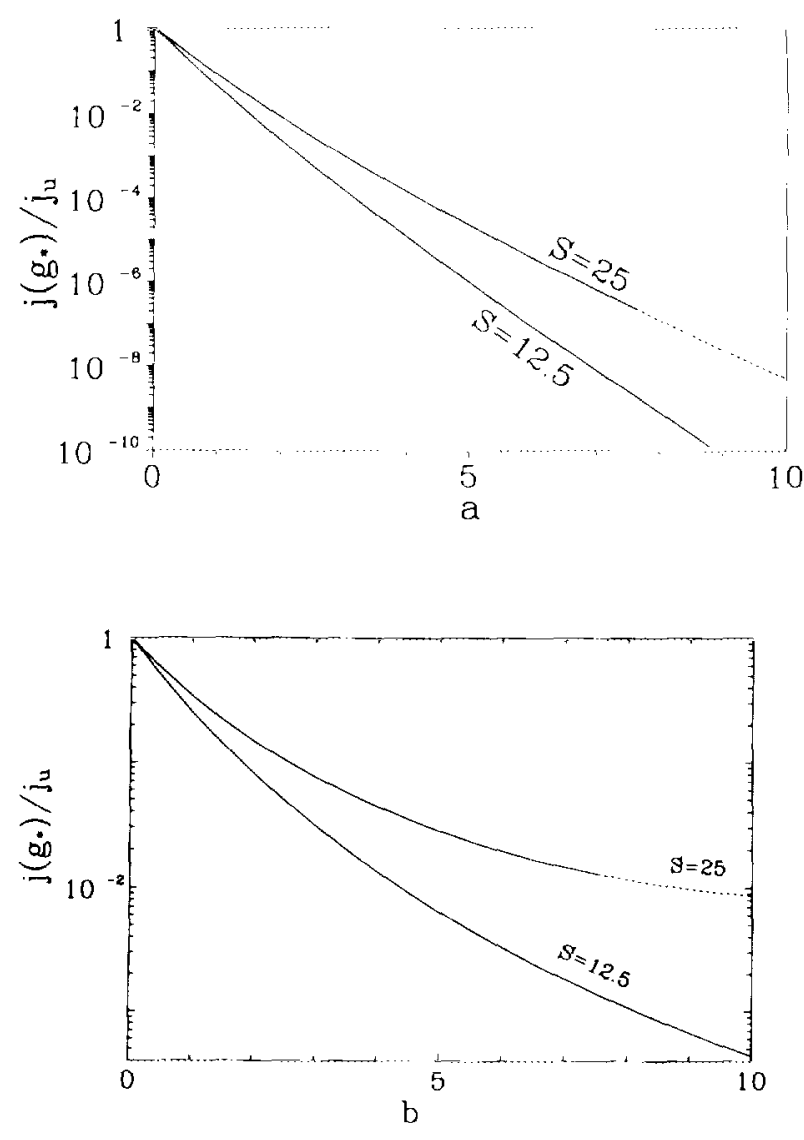

FIG. 4. Ratio between the rate of nucleation in presence of subcritical cluster loss in a diffusion cloud chamber and that in its absence (a) by diffusion $\left[j\left(g_{*}\right) / j_{*}\right.$, vs $a$ for $\left.\left.b=0\right)\right]$ and (b) by thermophoretic drift $\left[j\left(g_{*}\right) / j_{u}\right.$ vs $b$ for $a=0$ ].

diffusion on the nucleation rate in this case if we take $\phi=5$ as adopted by Becker and Reiss. ${ }^{2}$ The effect is found to be observable for equilibrium vapor pressures smaller than $10^{-}$Torr. At $p_{c}=10^{8}$ Torr, $a=1.8$ and $b=6.6$ for $S=12.5$. Thus, the onset of nucleation in this case would be affected by diffusion as well as by thermophoretic. Their magnitudes are roughly equivalent, as seen from Fig. 4. Our calculations therefore confirm qualitatively the previous results ${ }^{2}$ in which only the subcritical cluster loss only by diffusion was considered. It is also noted that the total pressure in the chamber is assumed at $1 \mathrm{~atm}$.

The diffusion cloud chamber is usually operated at a reduced pressure. The total pressure in a chamber is required to satisfy the condition ${ }^{17}$

$$
p_{c}\left(1-\frac{M_{1}}{M_{c}}\right)\left(1-\frac{T_{0}}{T_{1}}\right)^{\prime}>p_{1}>2.5 p_{c} .
$$

where $M_{1}$ is molecular weight of the vapor, and $T_{1}, T_{0}$ are temperatures at the condensing and evaporating surfaces. The upper bound for $p_{\text {r }}$ is that at which the temperature gradient is balanced by a composition gradient in the vaporgas mixture thus the thermal convection is prevented. The lower limit is estimated empirically, for which results are supposed to be independent of the amount of carrier gas used. 
To maintain the difference between $j_{u}$ and $j\left(g_{*}\right)$ within a factor of $10^{2}$ requires $a<1$, i.e., $p_{c}>4 \times 10^{-4}$ Torr for the organic species considered above. In a similar way we require $b<1$, i.e., $p_{c}>1.1 \times 10^{-3}$ Torr. Here we have used $d_{1}=0.71 h, d_{2}=0.19 h$ and $h$ is the height of cell $4.23 \mathrm{~cm}$ as reported in Ref. $18 . d_{0}$ has been taken $0.1 \mathrm{~h}$ as experimentally estimated by Daniel et al. ${ }^{19}$ The resulting $\phi$ is about 4.32 and $\psi$ is about 0.25 . Also we have used the upper bound for $p$ given by Eq. (19). We conclude that the onset of homogeneous nucleation will begin to be affected by subcritical cluster diffusion and thermophoretic drift when $p_{c}<10^{-3}$ Torr. Under this condition, the subcritical cluster loss by thermophoretic drift becomes more important than that by diffusion.

It should be noted that the effect of diffusion and phoretic drift on nucleation does not limit to subcritical clusters loss. Nucleated particles, for example, can reach the upper surface before growing to a free fall size by action of phoretic forces at low pressures. Thus higher supersaturations are observed to maintain a same rate of nucleation. ${ }^{20}$

\section{CONCLUSION}

We have investigated homogeneous nucleation process of a vapor in a spatially inhomogeneous system by extending classical nucleation theory to include subcritical cluster loss by diffusion and thermophoretic drift. Approximate analytic expressions have been obtained for cluster size distribution and rate of homogeneous nucleation. The primary re- sults of this paper are those of Eqs. (16)-(18) which can be used to estimate the effect of subcritical cluster loss by diffusion and drift in a temperature field on the onset of homogeneous nucleation.

'N. A. Fuchs, The Mechanics of Aerosols (Dover, New York, 1989).

${ }^{2}$ C. Becker and H. Reiss, J. Chem. Phys. 65, 2066 (1976)

${ }^{3} \mathrm{G}$. Shi and J. H. Seinfeld, J. Colloid Interface Sci. 135, 252 (1990)

${ }^{4}$ G. Shi, J. H. Seinfeld, and K. Okuyama, Phys. Rev. A 41, 2101 (1990)

${ }^{5}$ K. Binder, Rep. Prog. Phys. 50, 783 (1987).

'F. C. Everstein, Philip Res. Repts. 26, 134 (1971)

${ }^{7}$ J. Bloem, J. Cryst. Growth 18, 70 (1973).

${ }^{k}$ J. U. M. S. Murthy, N. Miyamoto, M. Shimbo, and J. Nishizava, J. Cryst. Growth 33, 1 (1976)

${ }^{4}$ Z. M. Qian, H. Michiel, A. Van Ammel, J. Nijs, and R. Mertens J. Electrochem. Soc. 135, 2378 (1988).

${ }^{16}$ B. A. Scott, R. D. Estes, and J. M. Jasinski, J. Chem. Phys. 89, 2544 (1988)

"G. Turban, Y. Catherine, and B. Grolleau, Thin Solid Films 60, 147, (1979)

${ }^{12} \mathrm{C}$. W. Pearce, in VLSI Technology, edited by S. M. Sze (McGraw-Hill, New York, 1983).

1: W. G. Breiland, P. Ho, and M. E. Coltrin, J. Appl. Phys. 60, 1505 (1986) and references given therein.

${ }^{4}$ G. Hsu, R. Hogle, N. Rohatgi, and A. Morrison, J. Electrochem Soc. 1331,660 ( 1984).

${ }^{15}$ T. Furusawa, T. Kojima, and H. Hiroha, Chem. Eng. Sci. 43, 2037 (1988)

${ }^{16}$ C. S. Herrick and D. W. Woodruff, J. Electrochem Soc. 131, 2417 ( 1984).

${ }^{17}$ J. L. Katz, J. Chem. Phys. 52, 4733 (1970).

${ }^{18}$ C-H. Hung, M. J. Kranopoler, and J. L. Katz. J. Chem. Phys. 90, 1856 (1989); 92,7722 (E) (1990).

${ }^{19}$ C. F. Daniel, P. Ehrhard, and P Mirabel, J. Chem. Phys. 75, 4615 (1989)

${ }^{20} \mathrm{~J}$. Smolik and J. Vitovec, J. Aerosol Sci. 13, 587 (1982). 